\title{
CENTRO DE ALTO RENDIMIENTO PARA NATACION. MADRID/ESPAÑA
}

\author{
(HIGH YIELD CENTER FOR SWIMMING. MADRID/SPAIN)
}

José Miguel Pérez de Arenaza Barcelona y Joaquín Pujol Simón, Arquitectos

$151-42$

\begin{abstract}
RESUMEN
Obra en ejecución que desarrolla instalaciones deportivas cubiertas y al aire libre, destinadas a la natación en todas sus modalidades, así como al perfeccionamiento y enseñanza de la misma. De forma rectangular, consta fundamentalmente de tres niveles, el inferior destinado a instalaciones técnicas, el intermedio al desarrollo en planta de los distintos vasos y vestuarios, y el superior a espectadores.
\end{abstract}

En el centro de la planta y separando la zona cubierta de la de al aire libre se sitúan locales para la Federación Española de Natación $y$ de apoyo al complejo.

Edificio de depuradas lineas arquitectónicas adaptado perfectamente al solar en ladera, en el que tanto la estructura de gran luz, como la adaptación funcional al uso al que se le destina y la cuidada tecnología empleada en sus instalacines, harán de él en un próximo futuro; uno de los mejores en su género.

Esta previsto que esta instalación se use en los Campeonatos del Mundo de Natación del año 1986.

\section{SUMMARY}

Work in execution which develops open and covered sports installations, devoted to swimming in all its modalities so as to learning and training. Of rectangular shape, it is composed basically of three levels, the lowest devoted to technical installations, the medium one to the ground plan development of the different pools and dressing rooms and the upper level to the spectators.

In the center of the ground, and as a separation of the covered zone from the open one, the Spanish Swimming Federation and the administration buildings, are located.

Built in pure architectural lines, fully adapted to the ground slope, whether by the large span structure or its functional adaptation, and by the cared technologies employed in its installations, it will be classified as one of the best in its kind.

This installations is expected to be used in the World Swimming Chamships, 1986.
La construcción de este Centro Natatorio tiene su origen en la absoluta necesidad que tiene la Federación Española de Natación de poder disponer de un complejo de piscinas en la ciudad de Madrid, sobre la base de las siguientes consideraciones:

- Necesidad de disponer de un marco propio para poder llevar a la práctica las diversas fases de preparación de los deportistas de élite en las cuatro especialidades (natación, waterpolo, saltos y natación sincronizada), solventando de esta forma la dependencia que hasta hoy día ha tenido de otros organismos y entidades, lo que le ha supuesto, en muchas ocasiones, un déficit y una merma en los planes de preparación.
- Disponer para la mayoría de los clubs de natación de Madrid de un recinto para la práctica de la natación de competición, que hasta hoy día se ha desarrollado en piscinas municipalizadas, en las que ha debido compartir la lámina de agua con la práctica de la natación recreativa, lo cual les ha llevado a limitar considerablemente sus justas ambiciones deportivas.

- Disponer asimismo de unas instalaciones aptas para la celebración de aquellas competiciones nacionales e internacionales a las que una ciudad como Madrid puede optar. Como por ejemplo los Campeonatos del Mundo a celebrar en Madrid en agosto de 1986.

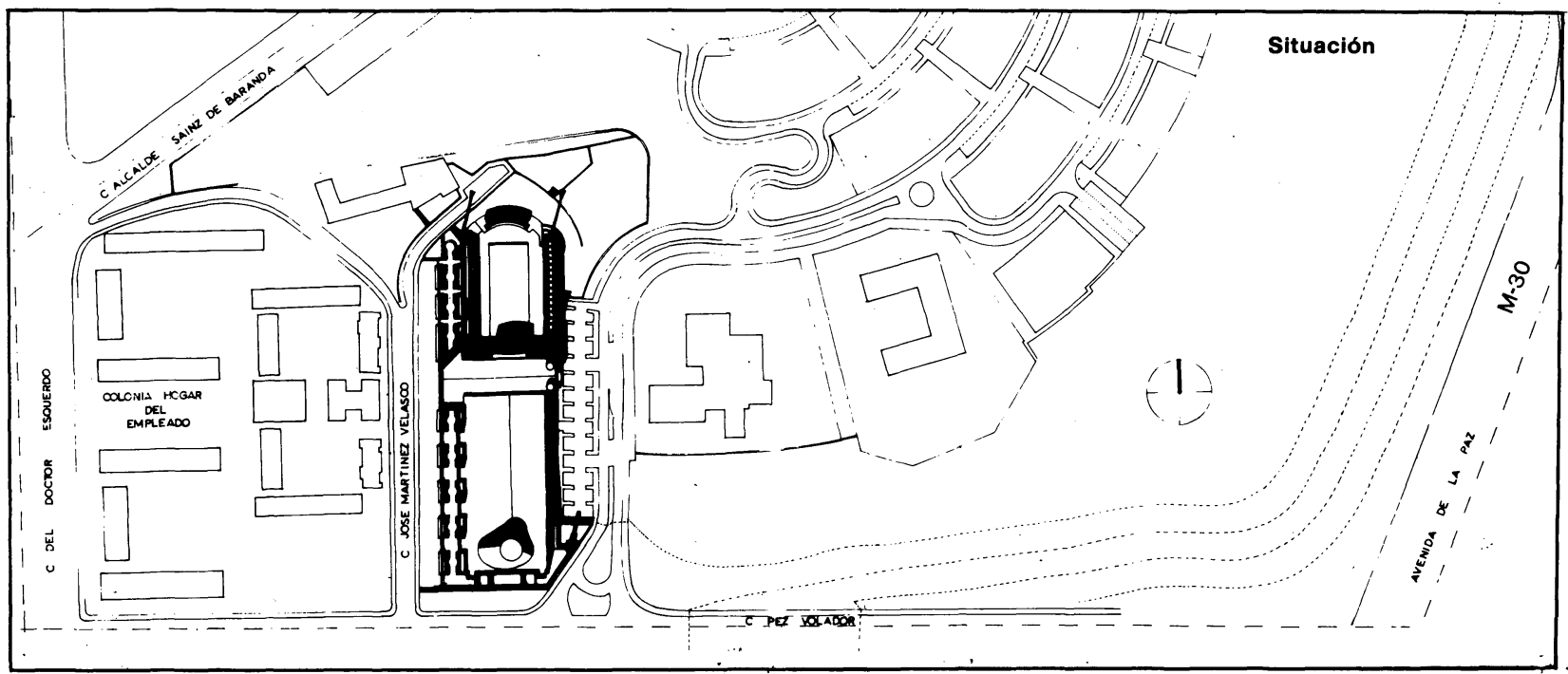




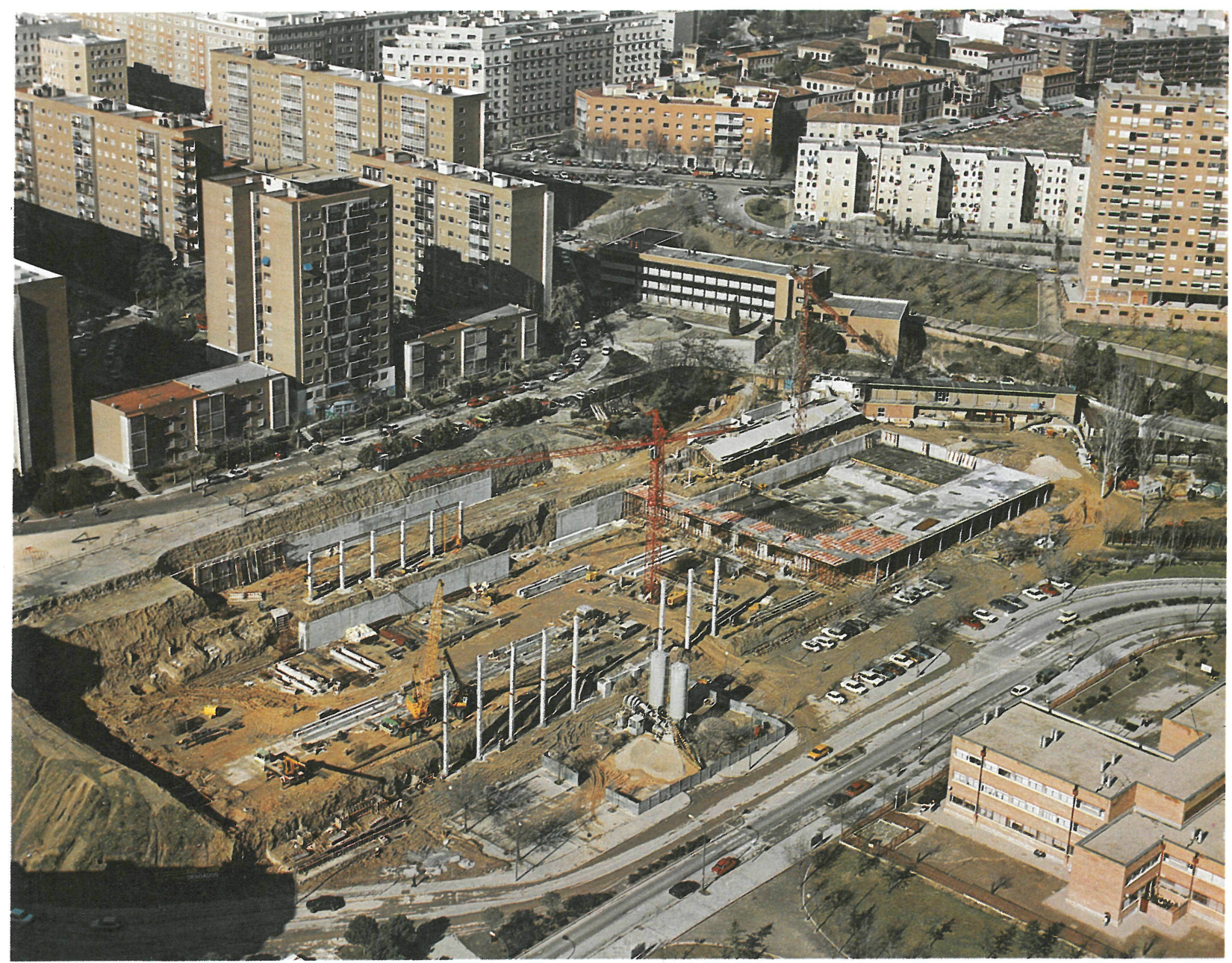

Estado de las obras -febrero 1985-.

El solar elegido para la construcción de este proyecto es una parcela existente en el Polígono 40 de la Avda. de la Paz de Madrid, cuya superficie es de $21.050 \mathrm{~m}^{2}$ y con la calificación de uso deportivo.

La elección se ha fundamentado básicamente en las características de fácil accesibilidad que presenta, dada su proximidad a las líneas de transporte urbano, la calle Doctor Esquerdo que transcurre a unos cien metros del solar.

El solar es de forma rectangular, orientado según su eje longitudinal en dirección N-S y con una topografía variable en la dirección E-O que va desde la cota 504,46 correspondiente a la fachada $O$, hasta la cota 494,70 que corresponde a la fachada $\mathrm{E}$.

Desde el punto de vista geológico, la parcela elegida está situada en una zona fronteriza entre los niveles inferiores de la "Facies Madrid» y las arcillas verdes conocidas comúnmente como Peñuela, formaciones ambas de depósito continental miocénico.

El análisis de los objetivos conduce a un claro planteamiento funcional: a) Posibilidad de uso de la instalación para grandes competiciones.

b) Posibilidad de uso de la instalación para uso cotidiano (entrenamiento, concentraciones, enseñanza, etc.).

Esta ambivalencia funcional plantea el problema de la convivencia de las grandes superficies y volúmenes, que habitualmente son requeridos por las grandes competiciones, con las necesidades funcionales que genera el entrenamiento y la práctica diarios. La resolución de los problemas que conlleva este antagonismo ha sido una de las preocupaciones fundamentales del proyecto.

Ha sido necesario por tanto, y así se ha planteado, lograr la compatibilidad de las dos funciones básicas del Complejo. Así pues, el proyecto contempla el dimensionado de espacios y superficies de uso diario que generarán los mínimos costos de mantenimiento y que a su vez pudieran incorporarse a las áreas y volúmenes requeridos para las grandes competiciones.

Atendiendo al planteamiento expuesto se elaboró conjuntamente con la Federación Española de Natación el siguiente programa de necesidades: 


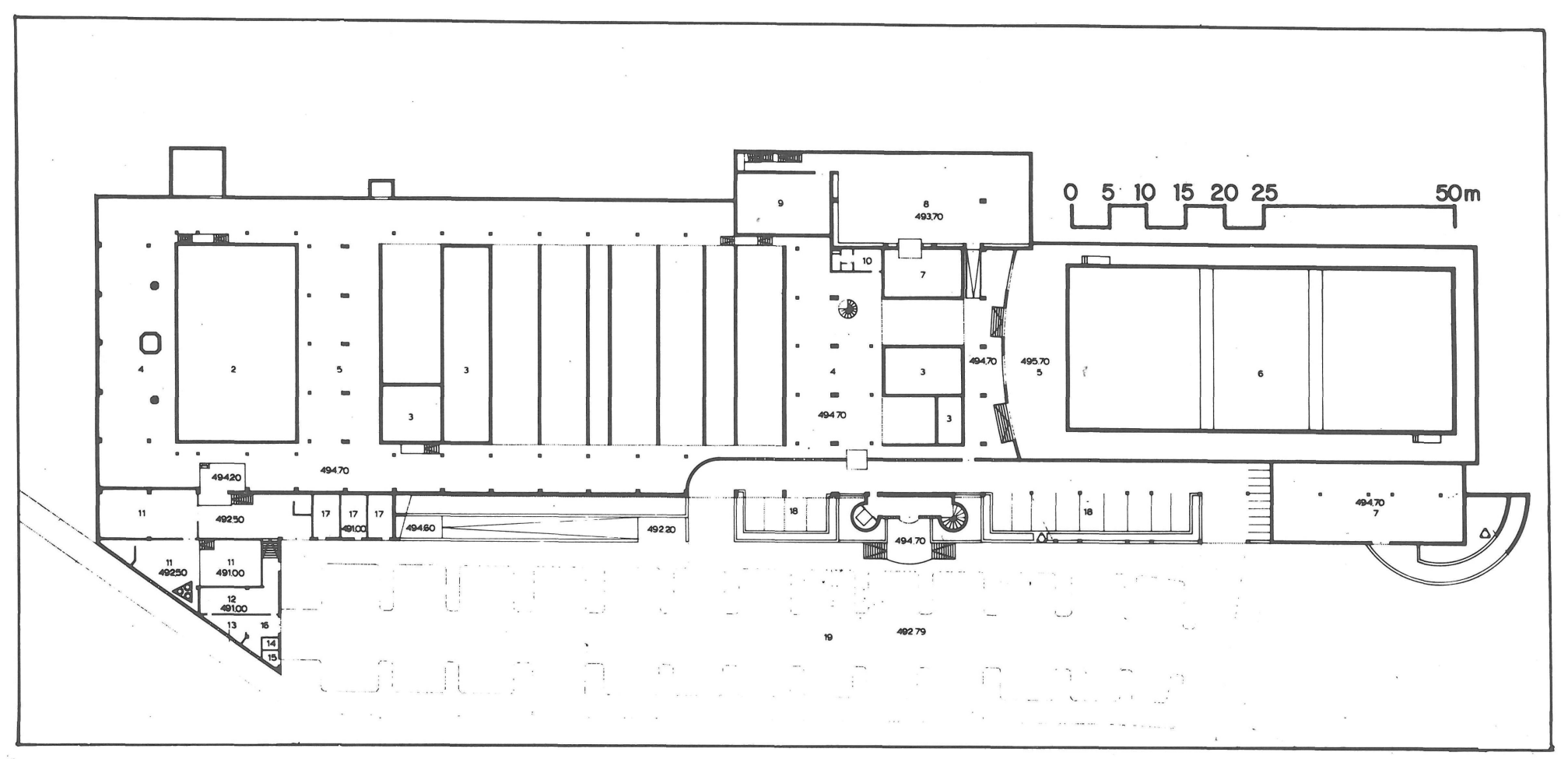

Planta de instalaciones

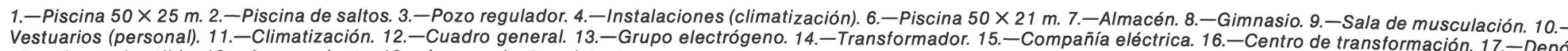

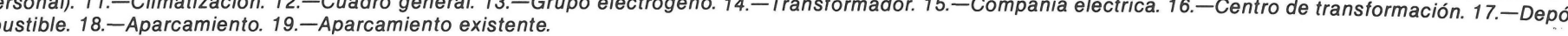

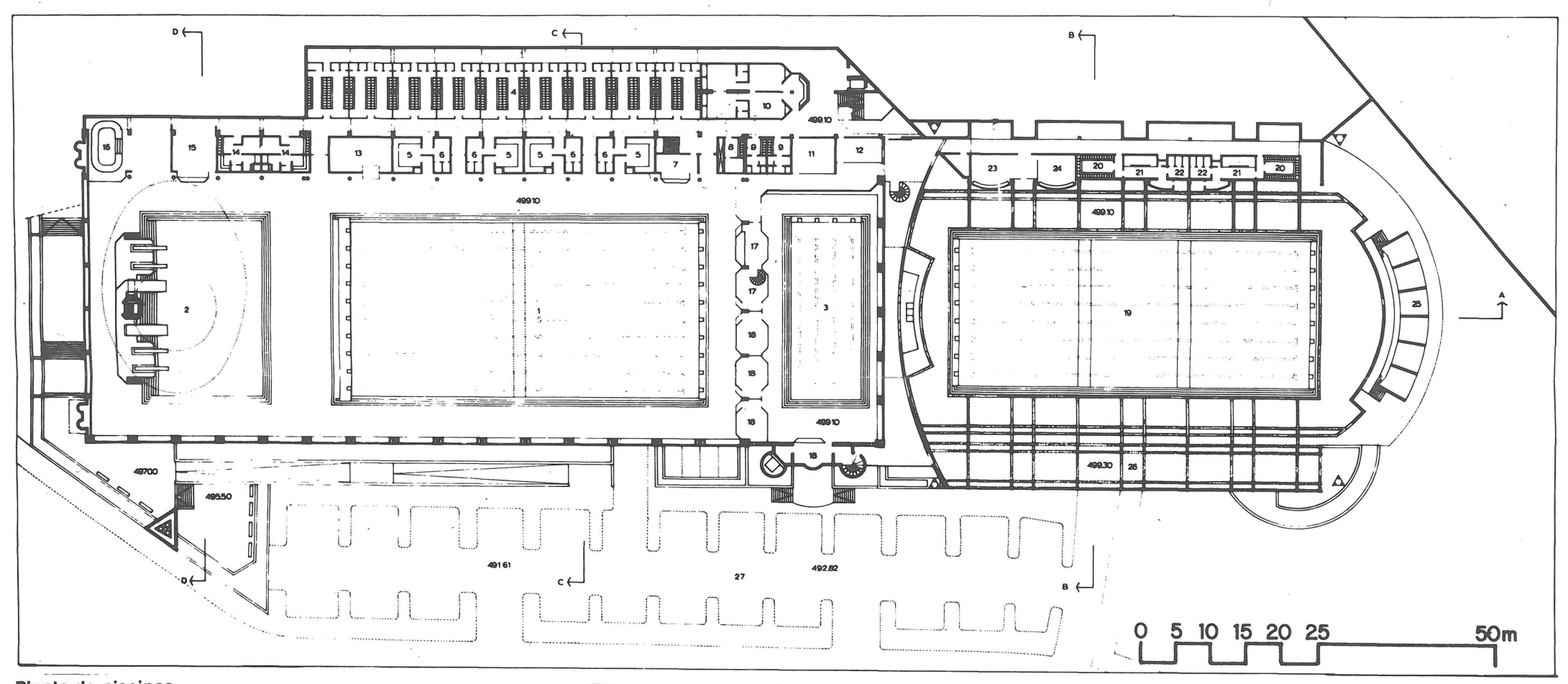

Planta de piscinas

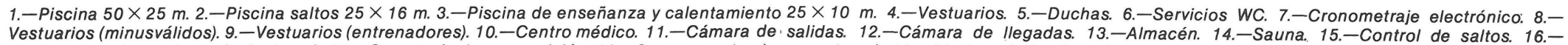

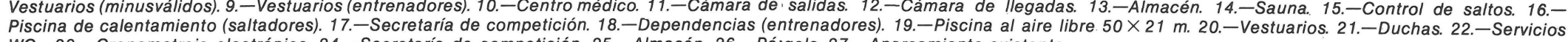
WC. 23.-Cronometraje electrónico. 24.-Secretaría de competición. 25. - Almacén. 26.-Pérgola. 27.-Aparcamiento existente.

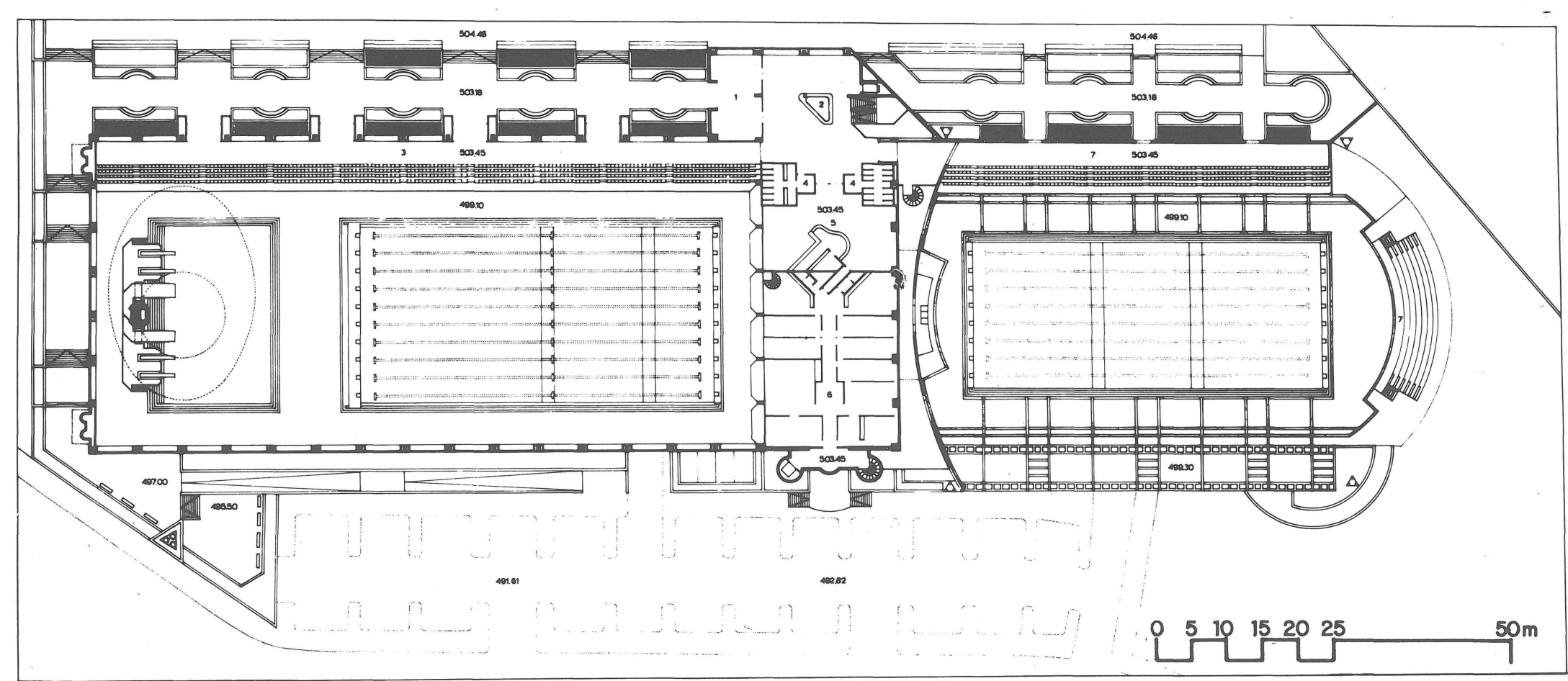

Planta de público

1.-Vestíbulo. 2.-Control usuarios. 3.-Graderíos. 4.-Aseos (público). 5.-Cafetería. 6.-Sede social (Federación Española de Natación). 7.-Graderío de piscina al aire libre. 


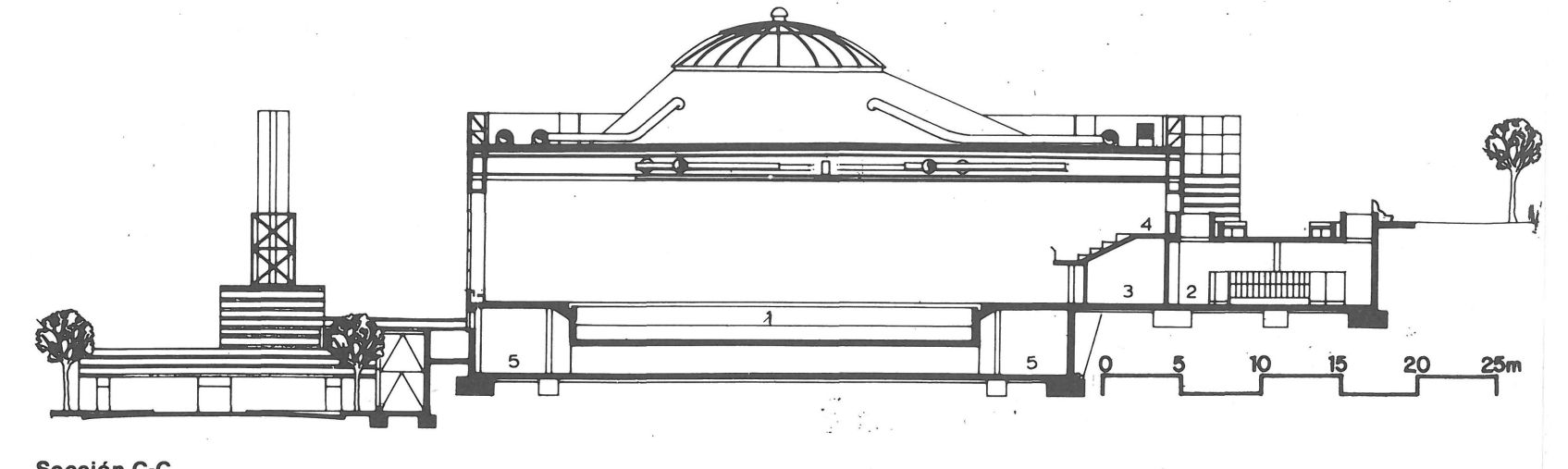

Sección c-c

1.-Piscina $50 \times 25$ m. 2.-Vestuarios. 3.-Duchas y aseos. 4.-Graderio público. 5.-Galerí de instalaciones.

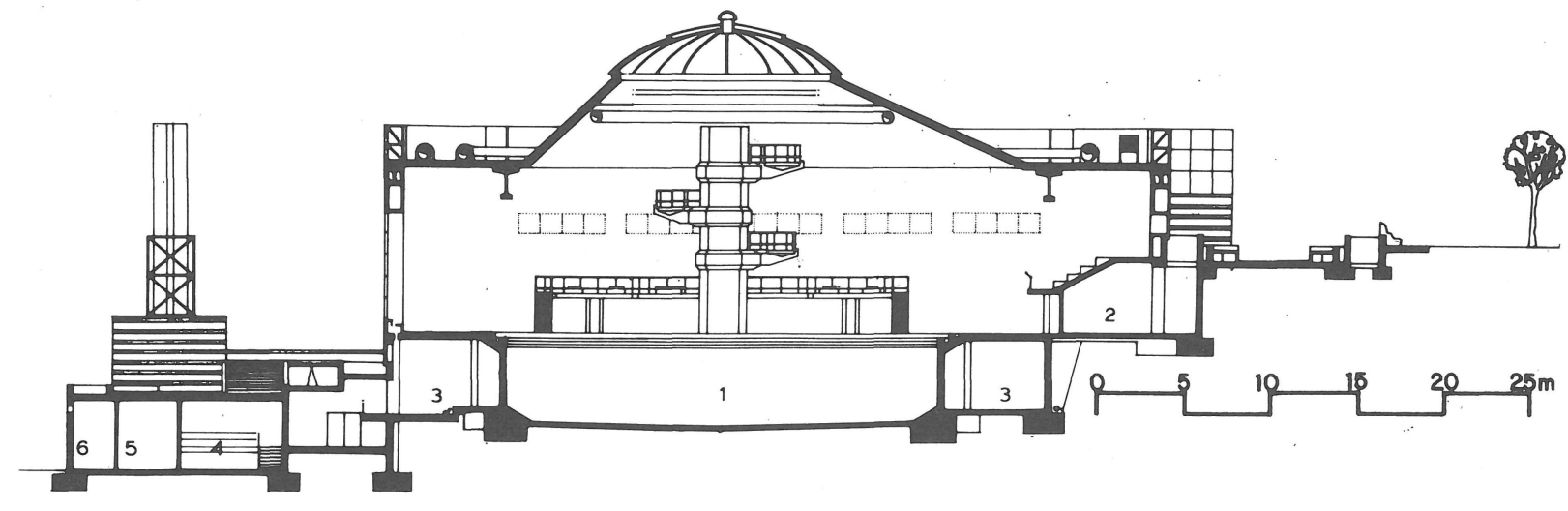

Sección D-D

1.-Piscina de saltos. 2.-Saunas. 3.-Galería de instalacines. 4.-Climatización. 5.-Cuadro general. 6.-Grupo electrógeno.

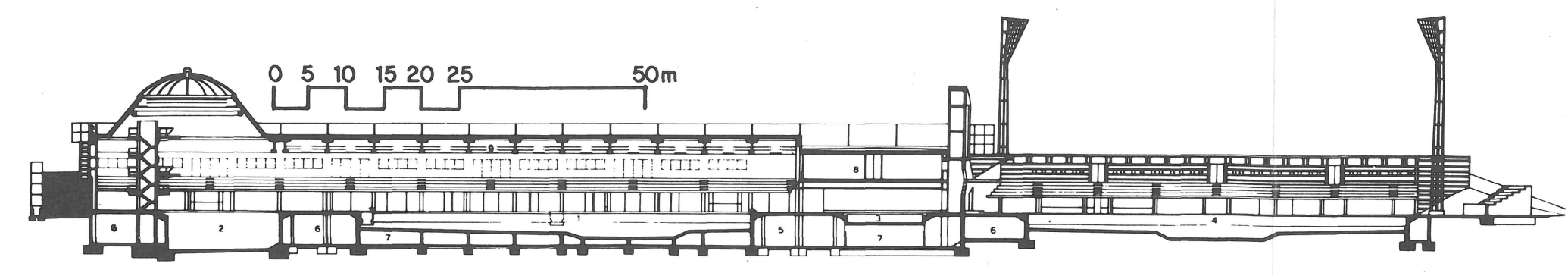

Sección A-A

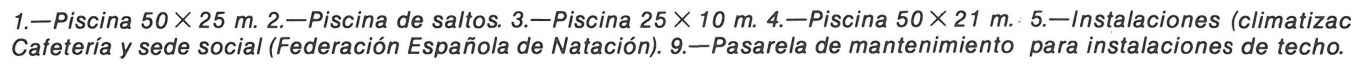

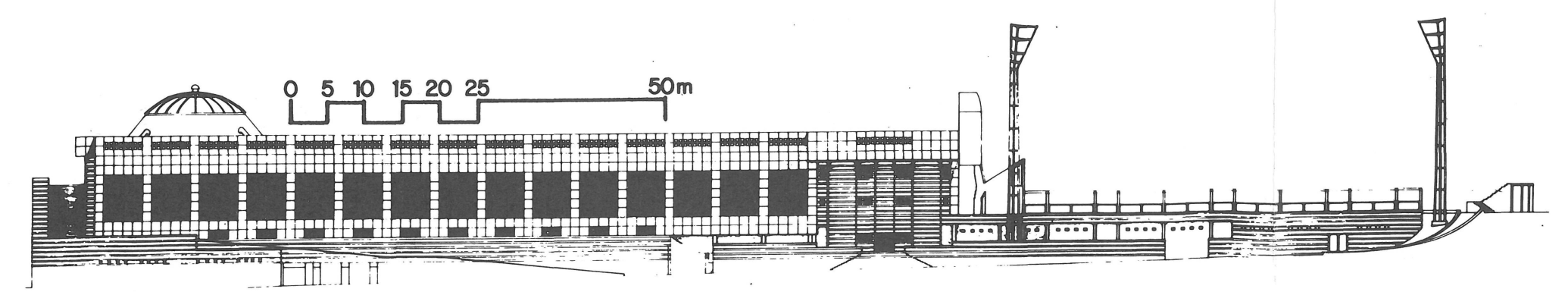

$$
\text { Alzadoc }
$$

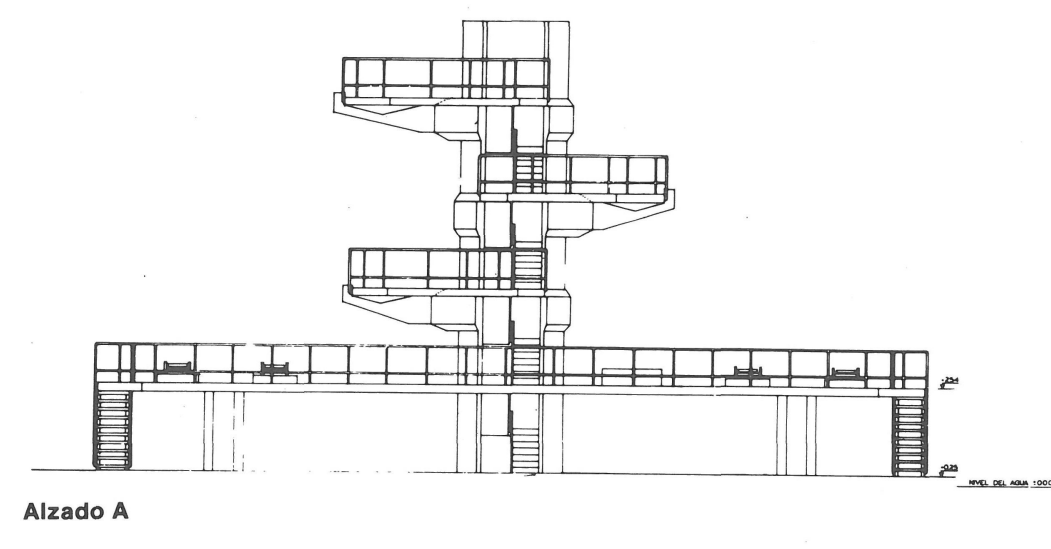

. $1 P$

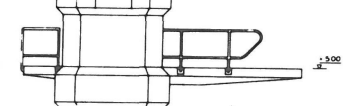
$\frac{1 P}{1}$ Alzado D

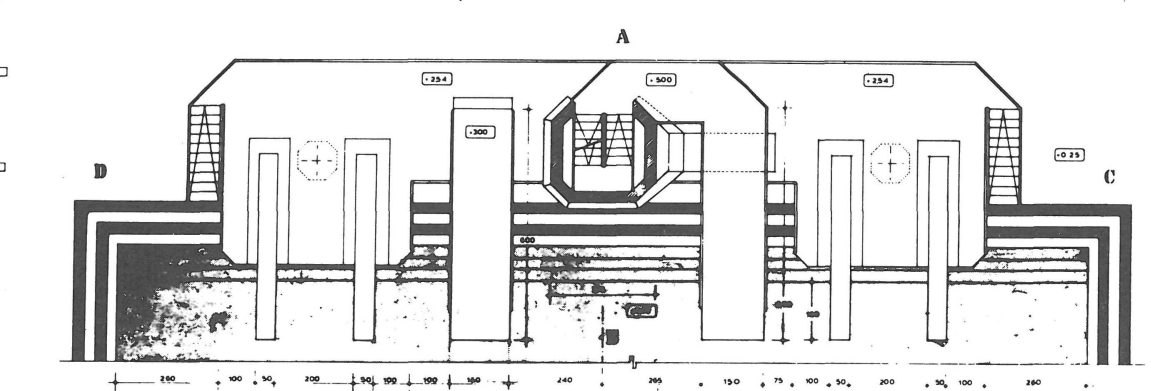
Planta nivel palanca $5,00 \mathrm{~m}$
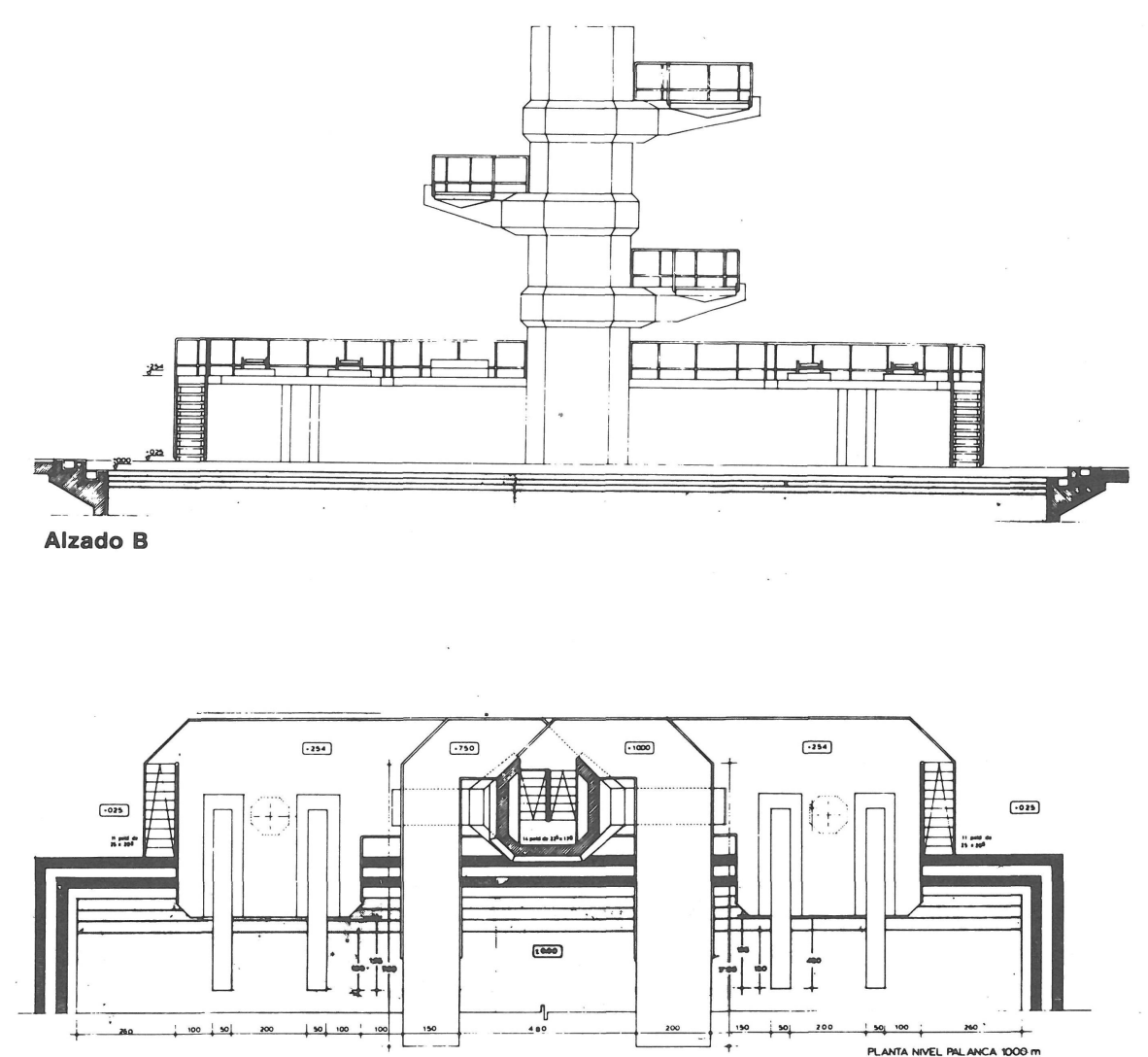

Planta nivel palanca 10,00 m 


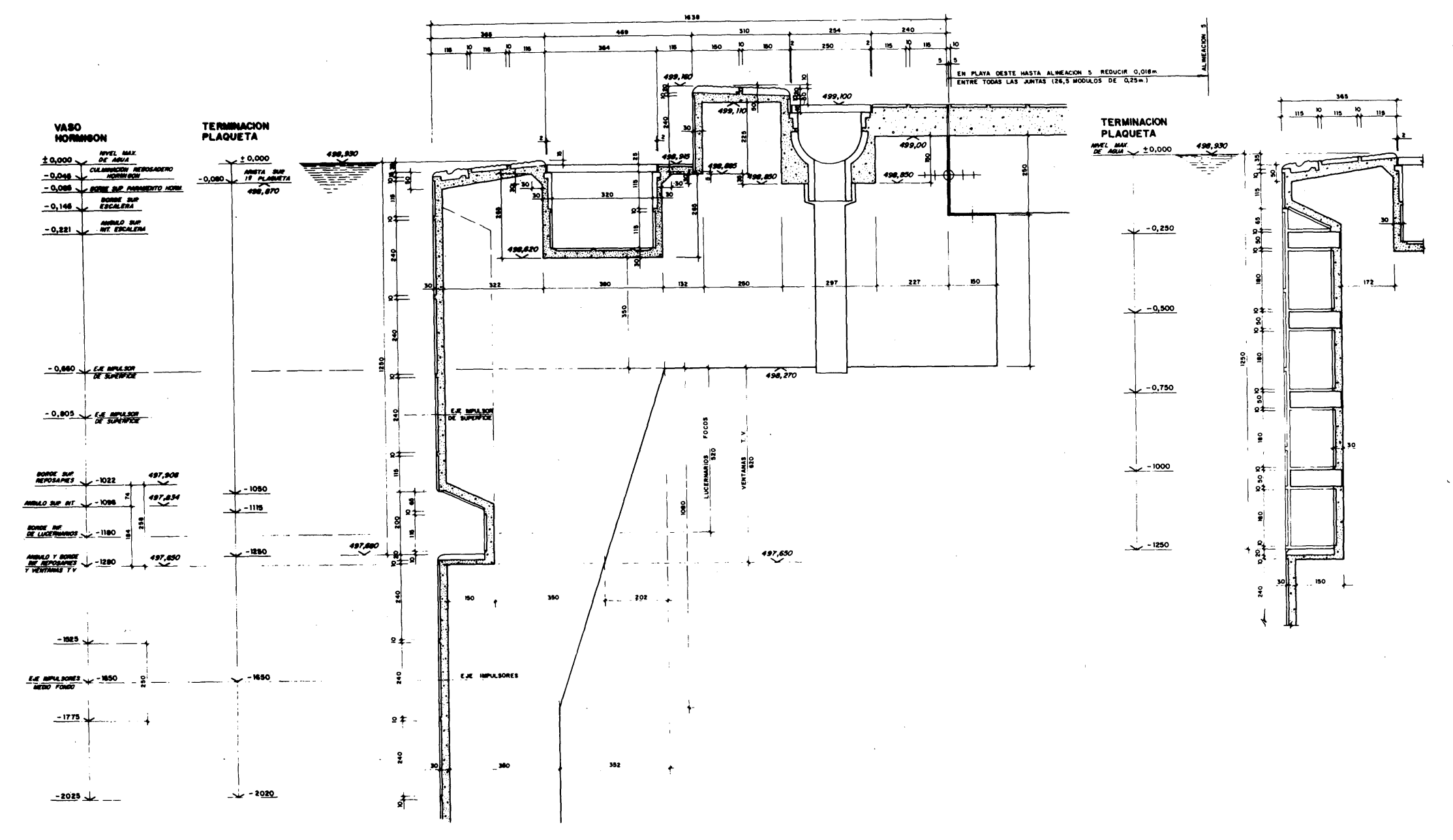

Vaso piscina descubierta y rebosadero. 
34

\section{Piscina cubierta de $50 \times 25$}

Esta piscina debe atender fundamentalmente a las necesidades de la práctica invernal. La anchura de 25 metros responde a un intento de versatilizar al máximo el uso de la superficie de agua sobre la base de poder ser utilizada transversalmente. A ello hay que añadir el apoyo aportado por una pasarela móvil que al deslizarse por encima del agua permitirá el uso absolutamente diferenciado en dos sectores de la misma piscina.

La profundidad de la piscina será de 2,00 metros en los extremos y de 3,00 en el sector central uniforme y podrá ser por tanto utilizada para cualquier tipo de competición.

La consideración que se ha dado al público es casi testimonial, 595 espectadores, ya que los criterios de racionalidad en el uso y mantenimiento de la instalación, han prevalecido sobre el fenómeno del deporte-espectáculo. De cualquier forma siempre será posible aumentar la capacidad de público de la piscina mediante la instalación de graderíos provisionales, pero que en todo caso no hipotecarán la superficie de uso deportivo.

Durante los Campeonatos Mundiales será utilizada como piscina de calentamiento y entrenamientos.

\section{Piscina cubierta de saltos}

La especialidad de los saltos se enfrenta en nuestro país, que por otro lado ha generado varios finalistas en competiciones mundiales y europeas, con el grave inconveniente de no disponer de una sola instalación apta para la práctica de los saltos durante todo el año. Es por ello que se ha planteado la inclusión de una instalación cubiert dentro del programa de necesidades a fin de acabar cor este déficit, respondiendo asimismo a los requerimiento: de la FINA para la celebración de las competiciones di saltos dentro del Mundial-86.

La torre dispondrá de palancas de $10,00,7,50,5,00$ y 3,00 metros y cuatro trampolines de 3,00 metros.

\section{Piscina cubierta de $25 \times 10$}

Esta piscina se plantea fundamentalmente con el objetivo de cubrir el espacio de la enseñanza de la natación desde la edad más temprana, dotándola para ello de una profundidad adecuada. A su vez esta piscina será de utilidad durante la celebración de competiciones en la piscina cubierta de $50 \times 25$ al poder ser utilizada como piscina de calentamiento.

\section{Piscina al aire libre $50 \times 21$}

Dadas las favorables condiciones climatológicas de la ciudad de Madrid, se ha creído conveniente disponer de una piscina de entrenamiento y competición al aire libre con el fin de que pueda ser utilizada durante cinco meses al año previa climatización del agua. Esta piscina, al margen de generar más disponibilidad de lámina de agua, traslada la práctica de la natación a su entorno más favorable que es el aire libre.

Se ha pensado en ella para desarrollar las competiciones de natación de los Mundiales del 86, previa adaptación, mediante graderíos provisionales, de los espacios requeridos para el público ya que el número de localidades fijas que se plantean (594 asientos), es el que suele darse de promedio en nuestras competiciones, aunque a esta toma de decisión han intervenido también consideraciones formales y de adaptación al entorno.

\section{Gimnasio y sala de musculación}

Dado el carácter primordial que va a tener la instalación como Centro de Alto Rendimiento, se ha incluido dentro del programa de necesidades aquellos recintos como son gimnasio y sala de musculación, absolutamente necesarios para la formación total del nadador.

\section{Sede social de la FEN}

Dentro del programa de necesidades se ha previsto la reserva de un espacio que albergue la sede social de la Federación Española de Natación para que de esta forma quede ubicada en un lugar idóneo como es este edificio, hecho por otra parte más que justificado dado el carácter de centro neurálgico, que para toda la natación española va a adquirir este complejo natatorio.

La adaptación de este programa de necesidades a las posibilidades y características del solar es lo que genera la propuesta formal que se traduce en el presente proyecto. Atendiendo a la orientación del solar, así como a la forma sensiblemente rectangular y a la pendiente en sentido descendente hacia el Este del mismo, el programa de necesidades se ha desarrollado a través del eje longitudinal del solar, considerando la parte superior del mismo y a su vez próxima a la calle Dr. Esquerdo, como lugar de acceso al edificio, el cual se va escalonando y adaptando a la topografía del terreno.

Todas las dependencias cubiertas se han ubicado en un único edificio que se sitúa en la zona Sur del solar, relativamente próximo a las edificaciones existentes, reservando para la mitad Norte, más alejada de las edificaciones, el espacio destinado a la piscina al aire libre.

En el edificio se han diferenciado claramente los recorridos efectuados por los usuarios y los que realizan los espectadores. Los primeros, después de superar el control único que se sitúa en el vestíbulo general, situado en la cota 503,45, desarrollan toda su actividad en la planta de piscinas, situada en la cota 499,10. El público, sin embargo, no desciende nunca de la cota 503,45 con lo cual se evita la interferencia de circulaciones con los usuarios.

Las piscinas se desarrollan, como hemos dicho, según un eje longitudinal. La piscina de $50 \times 25$ y la de saltos se 
encuentran dentro del mismo espacio, mientras que la de $25 \times 10$ queda separada de las demás por unas dependencias que estarán destinadas a los entrenadores y a la secretaria de la competición.

La altura del techo de la piscina de $50 \times 25$ es de $7,90 \mathrm{~m}$ con respecto al nivel de la lámina del agua, mientras que en la zona de los saltos la cubierta se transforma en una cúpula con el objetivo de generar el mínimo volumen posible. De esta forma se cumple con los requisitos que la reglamentación sobre los saltos obliga en lo referente a distancias con respecto a los puntos de la cubierta. Esta solución garantiza a su vez la máxima visibilidad de los espectadores. La altura del techo de la piscina de $25 \times 10$ se sitúa en $3,40 \mathrm{~m}$ con respecto al nivel del agua.

En la planta de las piscinas se desarrollan todos los servicios anexos de vestuarios, duchas, aseos y almacenes. Asimismo, se han previsto vestuarios especiales para minusválidos que acceden a esta planta por medio de un ascensor que parte del vestíbulo de acceso. Hay igualmente vestuarios individualizados para los entrenadores, dos saunas y un centro médico asistencial. Se ha previsto un lugar destinado al cronometraje electrónico. Para los saltadores se ha habilitado una pequeña piscina de calentamiento situada junto al foso de saltos.

Los vestuarios y servicios anexos serán comunes para ambas piscinas a través de un acceso directo que se realizará entre los dos recintos.

La planta de piscinas se conectará directamente con la sala de máquinas, y a su vez con los locales de la FEN, a través de una única escalera que se sitúa en el espacio intermedio generado entre la piscina de $50 \times 25$ y la piscina de $25 \times 10$.

Desde la zona de vestuarios generales se conectará con el gimnasio y sala de musculación que se sitúan en la planta sótano y a la cota 493,70 .

Se preven asimismo unos espacios comunes para las piscinas cubiertas, y al aire libre para aquellas dependencias necesarias destinadas a competiciones, como son las cámaras de calentamiento, salidas y llegadas.

En la cota 503,45 a parte del vestíbulo general y los graderíos para el público, se sitúan los locales sociales de la FEN y un bar-cafetería. Ambos se sitúan en el forjado que sirve de cubierta a la piscina al aire libre. A los locales de la FEN se accede independientemente a través de la cota 494,70 del solar por medio de un núcleo de comunicaciones vertical formado por una escalera y un ascensor. Ambos permiten también el acceso hasta la cubierta del edificio desde la cual podrá accederse al interior de la piscina con objeto de proceder al mantenimiento de las instalaciones que transcurren por el techo. También la conservación del marcador electrónico se realizará por medio del acceso que permite la cubierta del edificio.

La planta sótano situada en la cota 494,70 se destina totalmente a las instalacines generales. Desde la misma se accede a la visitabilidad de los vasos de las piletas, lo cual permite el mantenimiento y control de las mismas, especialmente de todas aquellas instalaciones de depuración, así como de aquellas instalaciones especiales como son ventanas, focos y altavoces que están empotrados en los muros de las piscinas.

La piscina al aire libre se desarrolla longitudinalmente al eje principal del edificio. En ella se ubican algunos servicios anexos adicionales, como son aquellos espacios destinados al cronometraje electrónico, secretaría de competición y vestuarios para los jueces de la competición. Anexo a la playa se ha dispuesto de una pérgola con el fin de generar un espacio protegido de las radiaciones solares.

En el recinto al aire libre, y situado en la zona sur de la piscina coincidente con las salidas y llegadas de la competición, se ha diseñado una fachada curvada que, a modo de decorado escénico, protege de la vista directa sobre el edificio y culmina con el marcador electrónico. A su vez sirve también como telón de fondo de las ceremonias de proclamación de vencedores.

En la piscina al aire libre el acceso del público se realiza por la misma cota que al edificio cubierto, es decir, en la fachada Oeste. La disposición del graderío en la fachada Oeste asegura para el público una protección con respecto a los rayos solares especialmente en aquellas horas en que habitualmente se celebran las competiciones.

Situación: Calle José Martínez Velasco. Madrid

Promotor: Consejo Superior de Deportes. Comunidad Autónoma de Madrid. Federación Española de Natación

Dirección: José Miguel Pérez de Arenaza Barcelona, Joaquín Pujol Simón, Guillermo Ortego Carretero y Juan José Gómez Cuesta

Aparejadores: Enrique Granados Aumacellas, Ildefonso Pérez Gutiérrez, Luis Ortego Navarro y José Clemente Gómez de la Morena

Fecha inicio

Obra: $\quad 16$ de julio de 1984

Presupuesto

Contratación: 1.048 .641 .755 ptas.

Empresa

Constructora: Dragados y Construcciones

Fin Obra

previsto: Diciembre 1985 